\title{
Pédagogie et technologie : en marche vers « l'autrement »
}

\author{
Marcel Lebrun
}

Résumé Bien souvent, les outils technologiques nouveaux ne font qu'automatiser les anciennes pratiques : certains sites Internet d'enseignement ne font que reproduire les pratiques des salles de cours où l'information est transmise sans réel engagement cognitif de l'apprenant. Pourtant une valeur ajoutée à l'enseignement et surtout à l'apprentissage ne sera apportée que si ces outils sont intégrés dans des dispositifs qui respectent les nécessités d'un apprentissage de qualité : il n'y a pas de principes à l'apprentissage avec l'ordinateur qui ne soit pas simplement déjà un principe d'apprentissage. Sommes-nous sur la bonne route?

Mots clés Pédagogie - Technologie - Outils pédagogiques.

Summary Very often, the new technological tools are mere automation of old practices : some Internet websites simply reproduce traditional lectures where informations are given without any real cognitive involvement by the learner. But we know that we'll only get an added value for teaching and especially for learning if these tools are integrated in an effective learning environment: there is no principle in the experience of learning with a computer that is not already a learning principle. Are we on the good way?

Key Words Medical education - Technology - Technological tools.

Pedagogie Medicale 2000; $1:$ 45-53.

\section{Introduction}

C'est dans la foulée des rapports de la Conférence des recteurs européens ${ }^{1}$ et du rapport UCL- $575^{2}$, que l'on peut situer, aux environs des années 95, l'émergence d'une nouvelle dynamique pédagogique dans certaines universités européennes, en particulier à l'Université Catholique de Louvain (UCL). De manière générale, les discours initiateurs des milieux universitaires mais aussi économiques, sociaux et politiques parlaient tous d'une valeur à ajouter à un enseignement-apprentissage vu essentiellement comme une transmissionreproduction des " nécessaires " connaissances : compétences, attitudes, comportements, valeurs étaient les maîtres-mots. Face à cette demande pressante d'un renouveau pédagogique plus centré sur les qualités de la personne qui apprend (tête bien faite ou tête bien pleine ?), il fallait encore jalonner le chemin et mettre en place les mesures qui devaient permettre aux ensei- gnants et aux étudiants de le parcourir. A Louvain, de nombreuses mesures allaient être prises de manière à encourager, soutenir et évaluer les efforts des enseignants : la création d'un Institut de pédagogie universitaire et des multimédias (IPM) afin de veiller à la formation (dans toutes ses dimensions) des enseignants et à la valorisation de leurs initiatives, la création des "fonds de développement pédagogique " (FDP doté de 35 millions de francs Belges) afin de soutenir financièrement ces dernières, la création au sein de l'IPM d'une cellule multimédias assurant, avec le centre audiovisuel (CAV), le maintien des expériences acquises et l'assistance aux projets retenus par les FDP et finalement la mise en place d'organes d'évaluation des projets et de suivi de ces derniers.

Déjà nous soulignons l'importance de ces derniers points : l'expérience montre que la denrée la plus rare dans le développement des initiatives pédagogiques

Institut de pédagogie universitaire et des multimédias - Université catholique de Louvain - UCL/PM - Grand-rue, 54 B-1348 Louvain-la-Neuve - Belgique -Tél. : + 3210478927 - Fax : + 3210478939 - Courrier électronique : lebrun@ipm.ucl.ac.be 


\section{Nouvelles Technologies Educatives}

innovantes est le temps que les enseignants (écartelés entre leurs activités d'enseignement, de recherche et de service) peuvent y consacrer. Un soutien humain et financier s'avère utile pour qu'ils puissent trouver les ressources indispensables (humaines et matérielles); la nécessaire formation (aux niveaux pédagogique et technique) ainsi que le suivi et l'assistance ponctuelle doivent être organisés et rendus facilement disponibles.

On le voit, dans le cadre d'une impulsion institutionnelle forte, une attention et des attentes particulières étaient placées sur le développement d'outils bâtis sur les "nouvelles " technologies de l'information et de la communication (NTIC). Mais les démarches entreprises (par exemple, la création d'un institut " pédagogique " et "multimédias ") témoignent aussi de la volonté de ne pas séparer les efforts techniques des objectifs pédagogiques. Ainsi, comme nous l'avons montré, le développement d'outils multimédias pour l'enseignement et l'apprentissage ne porte ses fruits que dans le cadre de démarches pédagogiques innovantes construites autour de l'activité de l'apprenant, de la personnalisation des enseignements et des apprentissages, de nouvelles formes d'évaluation ${ }^{3}$.

\section{De l'intégration des technologies..}

Dans la plupart des discours sur le rôle des technologies dans l'enseignement et l'apprentissage, un raccourci de la pensée associe rapidement nouvelles pédagogies et nouvelles technologies... la rime est belle mais qu'en est-il dans les faits?

Une considération " historique » d'abord : dans leur intégration dans la vie sociale et dans les habitudes des individus, les technologies nouvelles ont d'abord copié (singé, diront les plus pessimistes) les technologies existantes; le cinéma a d'abord offert aux spectateurs des pièces de théâtre filmées, les informations de la télévision furent au début un simple "journal parlé ", les premières vidéos pédagogiques ressemblaient à des cours filmés, l'ordinateur soutint d'abord l'enseignement programmé.

"Autrement dit, nous répétons le passé, un peu comme si nous continuions à battre les tapis avec un tout nouvel aspirateur sophistiqué "

Adapté à notre contexte pédagogique, on peut constater que, au début, les nouvelles technologies adoptent les anciennes habitudes : des cours programmés à la publication de livres ou d'exposés magistraux sur
Internet, tel est le panorama qu'une vue rapide offre sur le développement pédagogique supporté par les technologies. Est-ce un "mal " nécessaire, un lieu de passage obligé ? C'est possible, mais force est alors de constater que pour atteindre les objectifs ambitieux visés, un soutien est indispensable pour que, sur ces bases, les promoteurs aillent plus loin en découvrant de nouvelles niches pour ces outils. Faut-il laisser faire ce développement "naturel " au risque que les promoteurs se contentent de la nouvelle vitrine qui fossilisera encore davantage les anciennes pratiques ?

Un problème d'antécédent et de conséquent ensuite : les recherches et analyses sur le rôle des outils technologiques dans l'enseignement et l'apprentissage sont relativement claires. Les nouveaux outils n'apporteront un plus à l'enseignement que dans le cadre de nouvelles pratiques pédagogiques, plus centrées sur la personne de l'étudiant, sur le développement de ses compétences (au-delà de l'accumulation des connaissances), sur l'interaction relationnelle des êtres qui, tour à tour enseignants et apprenants, peuplent le triangle pédagogique. C'est la conclusion de l'excellent livre de M. Grabe et C. Grabe "Integrating technology for meaningful learning" qui nous indique que:

"Souvenez-vous de ceci : dans la plupart des cas, un enseignement efficace avec le concours des technologies est d'abord un enseignement efficace quel que soit le moyen utilisé " ${ }^{5}$.

C'est aussi une conclusion d'une étude récente (juillet 96) menée par l'Union Européenne dans le cadre du groupe de travail "Logiciels éducatifs et multimédia »: "Le multimédia a démontré son efficacité pédagogique dans le cadre de nombreuses expériences pilotes. Son intégration dans les pratiques ne pourra cependant se réaliser sans que les approches pédagogiques innovantes trouvent une meilleure écoute institutionnelle et sociale. C'est ainsi qu'il trouvera sa place dans le cadre général de la mutation des systèmes éducatifs " ${ }^{6}$.

Plus radicalement encore, un groupe de penseurs américains dans leur manifeste pour un technoréalisme, déclare :

"Aucune innovation technologique, aussi impressionnante soit-elle, ne pourra mener à la révolution du système d'éducation préconisée par le président Clinton. L'enseignement est un art qui ne peut pas être reproduit par l'ordinateur ou par Internet " ?.

Si la représentation que l'on se fait de l'enseignant est celle d'une personne qui se contente de fournir les 
informations à celui qui apprend, alors la réponse à la question " l'ordinateur remplacera-t-il un jour l'enseignant ? " risque bien d'être affirmative. La véritable question nous est posée : allons-nous contribuer à la transformation de la société de l'information en celle de la connaissance, de la société de l'interaction en celle de la rencontre?

\section{Le dilemme est difficile :}

- risquer de décourager les promoteurs, séduits par les nouveaux outils technologiques, en leur demandant de réfléchir aux objectifs et aux méthodes pédagogiques avant d'imaginer ou de concevoir les ressources technologiques (celles-ci sont coûteuses en temps, nécessitent une prise en charge si ce n'est une révision d'année en année alors que les supports technologiques donnent une illusion du définitif) ;

- laisser faire les promoteurs (qui, pour la plupart, proposent une simple transcription du "déjà-là " sur de nouveaux supports) en espérant que les technologies joueront un rôle de catalyseur pour le développement de nouvelles pédagogies. Cependant, il nous semble que pour atteindre les objectifs soulignés plus haut, il sera nécessaire de les accompagner tout au long du processus, de la conception à la réalisation et plus loin encore à l'intégration des productions dans l'enseignement et à leur évaluation en termes d'apprentissage.

\section{La cellule multimédias de l'IPM}

La cellule multimédias de l'IPM est constituée de 4 personnes (dont deux à mi-temps) aux compétences complémentaires : Internet, réalisation de multimédias interactifs, programmation multimédias et aspects pédagogiques des outils technologiques (implantation des outils dans les dispositifs, conditions et évaluation des impacts pédagogiques, etc.).

La cellule multimédias de l'IPM adhère aux objectifs généraux de l'IPM (formation pédagogique, assistance et suivi des initiatives pédagogiques, mise à disposition de ressources et information) dans le domaine particulier des nouvelles technologies de l'information et de la communication (NTIC). En particulier, elle veille à assurer une bonne utilisation des outils-logiciels développés et une bonne conservation des compétences déployées au service des enseignants et à la transférabilité de ces dernières.

Au niveau de la formation, elle tente de concilier le mieux possible les aspects technologiques et pédago- giques en montrant comment ils se soutiennent et se catalysent mutuellement.

La première année de son existence fut essentiellement dédiée à la sensibilisation des enseignants à ces deux pôles. Les titres des formations organisées sont éloquents de "l'ABC du multimédia " à "Les technologies... outils pédagogiques». Parmi d'autres, ces formations furent reprises plus de cinq fois tant la demande fut importante. Les années suivantes, à la suite d'une analyse des besoins auprès des enseignants et à une prise en compte des demandes au travers des initiatives pédagogiques en gestation, le programme s'est à la fois diversifié et spécialisé sur les deux aspects mentionnés : des techniques particulières des multimédias et d'Internet aux ingrédients des méthodes pédagogiques actives qui se marient particulièrement bien avec les technologies. Le programme actuel est bâti sur une quinzaine de formations et autant d'ateliers ; certaines de ces activités (création de sites Web, publication et recherche documentaire sur le réseau) ont déjà été reproduites plus de six fois tant la demande est forte.

L'équipe multimédias de l'IPM manifeste une attention particulière au conseil, à l'assistance et au suivi des enseignants qui développent des initiatives pédagogiques ayant recours aux NTIC. Cela se traduit en une implication particulière et individuelle des membres de l'équipe par rapport à certains projets soutenus par les fonds de développement pédagogique. On peut dire que chacun des quatre membres de l'équipe, en collaboration avec le centre audiovisuel, suit de manière soutenue de un à deux projets multimédias ou Internet, ce qui représente un véritable challenge vu l'ampleur de certains projets. Nous mentionnons un multimédia interactif pour l'apprentissage autonome des méthodes de travail dans les études supérieures, un multimédia interactif d'apprentissage des techniques de sculpture de la pierre, un cédérom sur la recherche et l'analyse documentaire, un logiciel permettant l'apprentissage du droit des obligations, le développement d'un site Internet d'apprentissage de la philosophie, un atelier multimédia de réalisation de travaux personnels sur le thème "Mouvement et Santé ". Les questions pédagogiques sont omniprésentes de la conception de l'outil à son évaluation auprès des étudiants. Une part importante du travail est également dédiée à l'assistance des enseignants par rapport à des questions spécifiques, au choix des outils et des techniques, à l'utili- 


\section{Nouvelles Technologies Educatives}

sation du matériel, etc. Un poste de production multimédias, ouvert aux enseignants, leur permet de subvenir à certains besoins technologiques dans un cadre où ils peuvent recevoir immédiatement les informations nécessaires et toute l'assistance utile. Une salle de formation (laboratoire informatique) disposant de dix postes informatiques multimédias permet l'organisation d'ateliers pratiques.

\section{Un modèle pour développer des pratiques pédagogiques innovantes soutenue par les NTIC}

Sur le chemin qui nous conduit à des applications technologiques pour enseigner aux applications technologiques pour apprendre, il est important pour nous de rechercher les ingrédients qui facilitent l'apprentissage, et le rendent effectif ${ }^{8}$.

En analysant plusieurs auteurs et en tentant de rapprocher certaines caractéristiques du processus interactif de l'enseignement et de l'apprentissage, il nous est apparu que cinq grandes facettes pouvaient en être esquissées :

MOTIVER celle qui relève du contexte général et de l'environnement didactique ;

INFORMER celle qui relève des informations, des connaissances et de leur support, des médias ;

ANALYSER " celle qui relève des compétences de plus haut niveau (analyse, synthèse, évaluation, sens critique,...)*;

INTERAGIR celle qui relève du recours aux diverses ressources et en particulier aux ressources humaines disponibles;

PRODUIRE

celle qui relève de la construction personnelle ou de la "production" (nouvelles connaissances et compétences, nouveaux projets,...).

*Nous avons mis le sigle de la troisième catégorie "Analyser " entre guillemets pour signifier qu'il n'est que représentatif d'un ensemble plus large de compétences de haut niveau.
A posteriori, nous constatons que ces cinq facettes de l'apprentissage constituent également des articulants de certaines méthodes pédagogiques dites innovantes. Par exemple, l'apprentissage par résolution de problèmes repose sur les piliers suivants :

- une situation concrète " posant problème " est le point de départ de l'activité ;

- des ressources appropriées (experts, documents, banque de données,...) sont mises à la disposition des apprenants de manière à "instruire le dossier " ;

- des activités de haut niveau sont sollicitées de la part des apprenants (par exemple, cerner ou poser le problème (la question qui figure généralement au bas de l'exercice traditionnel n'est pas nécessairement donnée en PBL... quelque chose ne marche pas... mais quoi ?), entamer des démarches d'observation, d'analyse, de recherche, d'évaluation, de réflexion,...) ;

- l'intégration (et non la juxtaposition) des connaissances est favorisée : résoudre un problème impose des regards multiples, des approches souvent multidisciplinaires ou interdisciplinaires, d'éprouver différents liens entre les connaissances déclaratives ;

- des alternances entre des temps de travail en équipe (le décorticage du problème, le brainstorming initial,...) et des temps de travail individuel ;

- des formes variées d'évaluation (par leur nature et par leur position temporelle dans le processus) permettant à la fois la régulation du processus, l'examen du chemin accompli dans l'atteinte des objectifs et la considération du produit obtenu.

Sans prétendre à l'exhaustivité, ces cinq facettes nous semblent intéressantes pour mieux définir un dispositif pédagogique, pour évaluer une initiative pédagogique, pour analyser une production technologique. Si, dans les intentions, la première facette (motivation) est souvent considérée ou évoquée (les usages des technologies constituent un facteur motivant pour les étudiants et pour les enseignants : intérêt de la tâche, richesse potentielle des compétences à exercer, soutien de l'ordinateur dans la réalisation), nous constatons, dans les initiatives pédagogiques proposées, que la deuxième facette (information) constitue une porte d'entrée privilégiée : mettre un cours sur le Web, donner accès à des bases de données, à des animations, proposer une vue dynamique de modèles, etc. Le "I " des NTIC joue à fond son rôle. Dans d'autres projets, l'accent est mis sur la possibilité offerte par la communication et l'interaction médiée par ordinateur interposé : pouvoir 
poser des questions sur le cours (enfin ?), développer des travaux coopératifs, des échanges linguistiques, etc. C'est ici que le « $\mathrm{C}$ » des NTIC trouve sa place.

Tout cela constitue, certes, des conditions potentielles intéressantes pour activer l'apprentissage mais reste encore fortement ancré dans la sphère didactique de l'enseignement sans garantir qu'un apprentissage effectif ait lieu. Les deux autres facettes relatives à l'activité de l'apprenant (l'exercice de compétences de haut niveau et la production-construction de nouvelles connaissances) semblent encore rester, dans la plupart des cas, en dehors de la sphère d'action des nouvelles technologies.

Pourtant, les logiciels de simulation et de modélisation, des déjà " anciennes " technologies (que l'on pense un bref instant à l'EAO) semblaient prometteurs dans cet ordre d'idées : analyser le comportement d'un modèle, critiquer l'influence d'un paramètre, généraliser, synthétiser ou abstraire une série de comportements, construire une nouvelle compréhension des phénomènes, etc.

Nous pouvons également rapprocher nos cinq facettes (motivation et information, interaction, compétences et production), des modes que nous avons proposés dans notre livre déjà cité : le mode réactif et son emphase sur l'environnement, sur l'information fournie, sur la contextualisation... ; le mode interactif et son emphase sur la nécessité des relations sociales, du feedback...; le mode proactif et son emphase sur l'activité de celui qui apprend, sur l'exercice des compétences et leur démonstration...

Cette classification est assez naturelle. Elle correspond aux grands courants pédagogiques dont certains insistent sur le rôle de l'environnement (réactif) dans le faconnage du comportement (les behavioristes skinériens, par exemple), d'autres mettent l'accent sur la compréhension et le développement des mécanismes cognitifs (proactif) dans la construction des connaissances (les constructivistes piagétiens, par exemple), d'autres encore appuyent la nécessité d'une interaction entre les deux approches.

Une résistance particulière à ces développements (par exemple, la marche du réactif au proactif) peut se trouver dans le fait que les nouvelles technologies ont apporté un grand nombre de solutions génériques (et relativement simples à mettre en place) dans le domaine de la diffusion des informations et dans la gestion des réseaux de communication ; les solutions favorables à l'apprentissage restent particulières (un modèle en économie politique n'a que peu de choses en commun avec une simulation d'un fluide dans une canalisation) et difficiles à mettre en place (techniquement et pédagogiquement).

- Pédagogiquement, ces méthodes actives sont proposées depuis plusieurs décennies (pédagogie du projet ou par le projet, démarche de résolution de problèmes, étude de cas, apprentissage coopératif,...) sans vraiment faire une percée spectaculaire. Elles sont coûteuses en temps pour l'étudiant et pour l'enseignant, demandent une révision complète des rôles tenus par ces partenaires et du statut du savoir, nécessitent une formation longue et en profondeur. Leurs effets ne sont pas immédiats et elles nécessitent des dispositifs pédagogiques bien souvent inscrits dans la durée.

- Techniquement, si mettre une page sur le Web devient aussi simple qu'imprimer une page sur une imprimante laser (les connaissances du HTML ou du Postscript ne sont plus nécessaires), le développement d'applications réellement interactives nécessite encore et toujours des connaissances informatiques lourdes (langages de programmation, algorithmique complexe,...). Il est donc important que les cellules d'accompagnement pédagogique et technique fonctionnent la main dans la main en encourageant les projets des enseignants (même s'ils ne réalisent pas toutes les facettes de l'apprentissage dont nous avons parlé), en stimulant le plus loin possible la réflexion pédagogique, en développant des dispositifs qui donneront toute leur pertinence aux outils envisagés.

\section{En route vers l'autrement: quelques projets}

Comme nous l'avons laissé présager, la plupart des projets présentent des caractéristiques relatives à l'enseignement souvent, au sens le plus traditionnel du mot : présenter des informations riches et variées, des moyens d'accès à l'information sous ses multiples formes (textes, tableaux, images) dans un cadre que l'on veut le plus incitant et le plus motivant possible. L'interactivité est souvent limitée à l'interactivité fonctionnelle (boutons, menus défilants permettent à l'utilisateur de naviguer dans la structure, de se repérer, etc.). Cependant par rapport au cours traditionnel, inscrit dans l'instant et évanescent, les supports informatiques permettent de rendre plus objectifs, plus pré- 


\section{Nouvelles Technologies Educatives}

hensibles la structure et les contenus de l'enseignement et, plus loin, d'envisager des perspectives réellement novatrices. De plus, la présentation des matériaux sur l'écran demande bien souvent, pour les enseignants, une révision du contenu et des modes d'appréhension de ce dernier par les étudiants. Les cours y gagnent donc en clarté, en structure et en contextualisation. Ce n'est pas suffisant pour garantir un apprentissage de qualité mais c'est une porte d'entrée nécessaire... à condition, comme nous l'avons dit, que des structures d'impulsion et de soutien encouragent l'enseignant à aller plus loin et à migrer doucement du pôle enseignant vers le pôle apprenant.

Dans ce qui suit, nous voulons proposer aux lecteurs des exemples, certains illustratifs et prototypiques des modes que nous avons exposés ci-dessus (le plus souvent inspirés du monde médical), d'autres réalisés à l'UCL avec le soutien ou non des FDP. Ils sont présentés ici dans une gradation allant d'activités cognitives de l'apprenant relativement élémentaires vers d'autres plus développées : appliquer, exercer, analyser, produire, etc.

\section{Des projets pour " informer "}

C'est sur ce pôle que les projets sont les plus nombreux ; si l'information est la porte d'entrée principale de ces projets, d'autres portes ou d'autres développements restent possibles et nous les mentionnerons chaque fois. Bien souvent, les développements entrevus vont vers une possibilité pour l'apprenant de s'entraîner ou de vérifier ses connaissances et aussi vers une interaction facilitée et plus étendue avec les enseignants et les autres étudiants.

Au niveau prototypique, nous puisons dans le domaine médical, le site du CISMeF (Catalogue et Index des Sites Médicaux Francophones) qui s'adresse en priorité aux professionnels de la santé. Il s'agit ici d'un répertoire dont le contenu peut être qualifié de méta-information : Actes de congrès, matériel d'enseignement, répertoires et guides, tout cela est au rendez-vous à un clic de distance. Différents accès sont prévus : alphabétique, thématique, par type de ressource, etc.

http://www.chu-rouen.fr/cismef/

- Un site sur la maladie thromboembolique veineuse agréablement présenté, avec une structure simple et efficace et des illustrations du cours qui synthétisent le propos. Ce site est aussi soutenu par des sociétés pri- vées. On y trouve également un départ vers les pages du séminaire de médecine interne générale des cliniques universitaires Saint-Luc associées à l'UCL.

http://www.md.ucl.ac.be/entites/mint/intr/hainaut $/ \mathrm{m}$ te/dossiermte/malthrombembol.html

- Un cours d'histoire de la critique littéraire sur Internet présentant les transparents (Powerpoint) utilisés lors du cours ; une façon intéressante de rendre disponibles (en différé) les informations données au cours.

http://lettresenligne.fltr.ucl.ac.be/Encyclo/index.htm Les axes de développement que nous entrevoyons vont dans le même sens suivant : contextualisation, communication et interaction accrue,...

- Des éléments pratiques relatifs à un cours d'électronique bâti autour d'un fil rouge, la construction d'un radio-réveil. On trouve sur ce site toute une série de documents allant des transparents utilisés au cours aux notices techniques à utiliser lors des travaux pratiques ; il s'agit de documents parfaitement formatés avec la technologie PDF (qui a l'avantage de conserver la mise en page originale).

http://www.dice.ucl.ac.be/ -jdl/InfoCours/

Outre les éléments d'amélioration cités plus haut et relatifs à l'interaction, on souhaiterait y voir quelques modules d'animations et de simulations relatifs aux circuits proposés. C'est l'objet d'un futur FDP.

- Des exercices complémentaires à la théorie exposée, c'est ce que nous trouvons sur le site du cours "Logique et argumentation " de la faculté de droit.

http://www.student.drt.ucl.ac.be/Logique/1116home. htm

Les textes proposés aux étudiants et les remédiations sont aussi soutenus par des fichiers sonores qui ouvrent une toute nouvelle dimension par rapport à la lecture sur l'écran. Les exercices, relativement traditionnels par ailleurs, montrent le chemin vers des activités plus dynamiques, vers des modes plus proactifs d'enseignement.

- C'est par ces activités cognitivement plus dynamiques que le cours sur Internet " Hydraulique à surface libre " se distingue. Des animations réalisées par des applets JAVA permettent à l'étudiant de se représenter l'influence des divers paramètres qui contribuent aux écoulements.

http://www.gc.ucl.ac.be/hydr/enseignement/didacti/h ome.html

Des guides aux opérations cognitives devraient davantage accompagner le parcours des étudiants : Quel 
serait l'effet de... ? Que devrait valoir tel paramètre pour que... ? Imaginer une situation dans laquelle... ? Bref, une pédagogie du problème plus qu'une pédagogie de la démonstration du comportement de la théorie.

\section{Des projets pour « communiquer "}

Différents sites greffent sur la couche d'informations (plus ou moins contextualisées), des possibilités d'interaction : pouvoir poser des questions au professeur, soumettre un problème aux autres étudiants... Dans d'autres cas, les outils d'interaction (mails, forums, listes, chat) sont vraiment au cœur du processus en déployant pour les étudiants de véritables travaux de groupe (apprentissage coopératif).

Au niveau prototypique des nombreuses réalisations en ce domaine, nous présentons le site de l'ALFEDIAM, l'association de langue française pour l'étude du diabète et des maladies métaboliques. Il vise à améliorer la qualité des soins délivrés aux diabétiques et à diffuser les connaissances sur le diabète. Un forum (liste de discussions) réservé aux professionnels de la santé est proposé.

http://www.alfediam.org/

- Dans le cadre du développement des interactions médecins-patients, nous signalons le site Virtanes (The UCL acute medicine web site) qui propose une large place à l'accueil et aux informations à destination du public. Ces nouveaux modes d'interaction entre personnes (ce que nous avons appelé dans notre livre l'interaction relationnelle pour la distinguer de l'interaction fonctionnelle des menus et des boutons à cliquer) pourraient devenir un atout majeur des nouveaux outils.

http://www.md.ucl.ac.be/virtanes/intro.html

- Dans le projet "Learn-nett ", les étudiants futurs enseignants (il s'agit d'un cours d'agrégation, la préparation des futurs enseignants du secondaire) de plusieurs universités de la Communauté française de Belgique (à l'origine du projet) et de l'Union Européenne sont invités à se mettre ensemble, à choisir un thème et à préparer une activité pédagogique utilisant les NTIC.

Des ressources (articles, notes de cours,...) sont à disposition ; des valves, un forum constituent des lieux de rencontres et d'échanges; un tuteur est assigné à chaque groupe de 4 ou 5 étudiants. Les productions des étudiants sont "affichées " sur les pages des groupes qui contribuent également au journal "Learn-nett».
Il s'agit ici d'une expérience qui équilibre relativement bien les cinq pôles dont nous avons parlé : Motiver (le choix du thème, apprendre à utiliser les technologies plutôt que de recevoir un exposé sur les supports technologiques), Informer (les ressources humaines et matérielles), "Analyser " (les compétences de haut niveau exercées), Interagir (technologiquement par les outils et cognitivement face aux apports des autres) et Produire (le dispositif créé, la contribution au site Web et au journal).

Il s'agit d'une expérience qui, aux dires des étudiants et des enseignants, est lourde à préparer et à conduire. Caricaturalement, on peut dire que si, comme enseignant, " $j$ 'en avais fini avec mes deux heures de cours par semaine, dans cette expérience, comme tuteur, j'ai l'impression d'être perpétuellement harcelé par les étudiants ».

http://www.ipm.ucl.ac.be/learnet/

\section{Des projets pour « apprendre »}

En bonne continuation de l'expérience qui précède, nous avons ajouté cette catégorie pour souligner des projets plus axés encore sur l'activité des étudiants, sur l'acquisition de compétences plus que de connaissances, sur les rôles nouveaux tenus par les professeurs et les étudiants, sur les possibilités des outils dynamiques comme les modélisateurs ou simulateurs.

Au niveau prototypique, nous citons le site VIPS (Virtual Internet Patient Simulation) qui permet (aux dires de la page d'accueil) d'entraîner les médecins comme des pilotes de ligne. Plus sérieusement, il s'agit ici de la mise en oeuvre d'un " exerciseur " qui vise le développement du processus de raisonnement clinique. Pendant une session VIPS, l'utilisateur est confronté à un cas clinique on line. Comme dans une consultation réelle, l'utilisateur pose des questions (en langage naturel), examine le patient en choisissant ses outils (yeux, mains, stéthoscope), demande des examens simples, dont le résultat est obtenu immédiatement, puis prend une décision. Le programme interprète le vocabulaire de l'utilisateur, répond aux questions, donne le résultat de l'examen physique ou des examens de laboratoire, fournit le résultat des décisions de l'utilisateur lors de la consultation suivante. http://www.swissvips.ch/

Au niveau illustratif, nous citons aussi le site du CIDMEF (Conférence internationale des doyens des facul- 


\section{Nouvelles Technologies Educatives}

tés de médecine d'expression française) http://www.cidmef.u-bordeaux2.fr/ dans lequel la rubrique "Apprendre " propose différents cours en ligne et des liens vers des simulations de haut niveau comme le "Virolab ", une simulation attrayante d'un laboratoire de biologie

http://cmusun29.unige.ch/apprentissage/virolab/.

- Dans son cours "Mouvement et Santé ", un professeur a décidé de mettre à la disposition des étudiants, non pas un " cours" plus ou moins interactif sur le domaine, mais les outils (programmes de développement de multimédias) qui permettent de construire des multimédias afin que les étudiants réalisent en groupe une véritable "élocution multimédia " en lieu et place du traditionnel travail personnel. Après une "brève " initiation aux outils, il s'agissait de repérer ou de construire les médias (images, vidéos, textes) et de construire une structure arborescente interactive que d'autres étudiants pourraient consulter. On remarque que les cinq pôles que nous avons proposés sont relativement bien représentés dans cette initiative. Au-delà des difficultés techniques (apprentissage du logiciel, réalisation des médias), une constatation principale fut la difficulté pour les étudiants de sortir de la pensée linéaire (une page permettant d'accéder à une autre page) pour construire une véritable architecture mettant en évidence la relation entre les concepts, entre la théorie et la pratique; il s'agit d'une manifestation de la difficulté de construire le résultat d'un apprentissage effectif (la carte conceptuelle des savoirs) tout en apprenant...

Il n'y a pas de références web à cette activité : l'activité majeure se passe dans la salle de travaux, entre les étudiants "autour " et non dans l'ordinateur : l'interactivité relationnelle marche à fond.

\section{Vers une intégration...}

Comme de nombreuses catégorisations, celle qui est proposée ci-dessus ne doit pas être prise comme un armoire dont les tiroirs serviraient à ranger les initiatives pédagogiques. Il s'agirait plutôt d'axes ou de composantes qui permettraient de définir, de qualifier l'objet en question. De nombreuses expériences (parmi celles que nous avons décrites d'ailleurs) mélangent les modes proposés : le texte du cours avoisine les diapositives de l'exposé et est accompagné d'exercices autocorrigés et d'un outil de communication.
Des outils permettent d'ailleurs aux enseignants de créer relativement facilement et dans un environnement intégré les " cours en ligne " qu'ils souhaitent. Les programmes auteurs comme Topclass ou WebCT (choisi à l'UCL) permettent de créer ces sites " d'enseignement sans distance " sans devoir recourir à plusieurs outils différents.

Au niveau prototypique dans le monde médical, nous illustrons notre propos par le site ALPES MED de l'université Joseph Fourier de Grenoble qui propose (sur la base du logiciel Topclass) un environnement d'autoévaluation des étudiants en médecine.

http://www-sante.ujf-grenoble.fr/sante/ (utilisateur et mot de passe "visiteur»)

- A l'UCL, un tout nouvel environnement de développement de cours en ligne a été mis en place autour du logiciel WebCT. Les premiers projets sont annoncés pour l'année académique 2000-2001.

http://www.icampus.ucl.ac.be/

\section{Les difficultés pour les enseignants}

Des difficultés d'ordre pédagogique d'abord : dans le voyage vers "l'autrement " (une pédagogie plus centrée sur les activités cognitives de haut niveau de l'apprenant), des enseignants volontaires mettent en place des dispositifs (méthodes et outils) qui permettent les approches pédagogiques innovantes. Parfois, les projets pédagogiques supportés par les technologies conservent la trace de la communication unidirectionnelle des exposés magistraux des salles de cours. Le fait que des boutons et des menus "interactifs " agrémentent les publications sur Internet ne change pas grandchose ; ils permettent seulement l'accès à l'information ce qui ne garantit nullement l'apprentissage, la transformation de l'information en connaissances et en compétences.

Cependant, le fait d'accorder que le «savoir " soit délivré par un média technique (moi, l'enseignant, ne suis plus le "seul gardien du savoir») et que l'apprenant puisse y accéder directement (il peut accéder seul au " temple du savoir ") accorde déjà une place nouvelle à son implication et libère l'enseignant des tâches banales et répétitives pour qu'il puisse se consacrer à des rôles plus nobles (non réductibles technologiquement); ce chemin doit être guidé et c'est là une action majeure de l'IPM.

Le manque de temps est souvent mis en avant dans les 
difficultés ressenties par les enseignants : temps de développement des nouveaux outils, temps à consacrer aux étudiants dans la préparation et la conduite des nouveaux dispositifs qui les encadreront afin que les outils manifestent le plus d'efficacité possible. Plusieurs pistes sont lancées à l'UCL : la possibilité, dans le cadre des FDP, de l'engagement d'un collaborateur qui, soit permettra la continuation de l'enseignement pendant que le promoteur développera ses nouveaux outils et dispositifs, soit lui donnera le coup de main direct nécessaire à la réalisation de son projet. La cellule multimédias peut aussi l'aider considérablement en mettant son expertise technique et pédagogique à sa disposition.

Comme nous l'avons vu, la réalisation de ces projets nécessite bien souvent la contribution d'experts d'horizons différents et le travail en équipe. Il s'agit là aussi de difficultés qui peuvent être partiellement levées par les ressources humaines de l'IPM et par l'encouragement et le soutien qu'il offre dans le cadre de la révision et de l'évaluation des programmes de cours. Une réflexion sur une meilleure organisation des cours, des travaux pratiques, des séminaires, souvent dévolus à des personnes différentes, est aussi nécessaire dans cet ordre d'idées.

En conclusion, nous pouvons dire que la pédagogie à l'UCL est en marche vers "l'autrement". Institutionnellement le message pour une pédagogie plus centrée sur l'apprenant est claire, les soutiens concrets à ce développement sont en place (l'IPM, sa cellule multimédias, le soutien des FDP). Les enseignants se tournent de plus en plus vers les nouvelles technologies en franchissant le premier pas de la dévolution de l'apprentissage sur l'étudiant. Les efforts doivent être maintenus afin que les NTIC jouent leur rôle catalyseur pour le développement d'approches pédagogiques réellement innovantes.

\section{Références}

1. The European Round Table of Industrialists. Une éducation européenne. Vers une société qui apprend. Bruxelles :Publications ERT, 1995.

2. Groupe "UCL 575 ", Université Catholique de Louvain, Juin 1995.

3. M. Lebrun. Des technologies pour enseigner et apprendre. Bruxelles :De Boeck,1999.

4. In other words, we end up automating the past, as we continue beating the rug with a fancy, new electric vacuum cleaner. D.M. Gayeski. Multimedia for Learning. New Jersey: Educational Technology Publications, 1993.

5. Just remember: in most cases, effective teaching with technology is effective teaching by any means. Grabe, M. \& Grabe, C. Integrating technology for meaningful learning. Boston : Houghton Mifflin Company, 1996.

6. Commission Européenne. Rapport du groupe de travail "Logiciels éducatifs et Multimédia ". Document de travail des services de la Commission. Document SEC(96). $1996 ; 1426$. http://www2.echo.lul

7. Sheck D, Shapiro A, Johnson S. Technorealism: A Manifest From A New Generation of Cultural Critics. 1998. http://www.technorealism.org (traduction de Bibeau R).

8. Lebrun M.Des technologies pour enseigner et apprendre. Bruxelles : De Boeck,1999. 